\title{
BER Assessment of Conventional QOSTBC3, RAR- QOSTBC3 and Taha3 Space-Time Coding Techniques over Diverse Channels
}

\author{
Shyna Lavish Kansal* \\ School of Electronics and Electrical Engineering, Lovely Professional University, Phagwara - 144411, Punjab, India; \\ shynachugh2@gmail.com, lavish.15911@|pu.co.in
}

\begin{abstract}
Even with the presence of complex entities in their code structure, Conventional Quasi-Orthogonal Space-Time Block Codes (QO-STBC) can accomplish the goal of obtaining full rate but with the drawback of higher decoding complexity and less diversity gain. The logic behind more complications while decoding quasi-orthogonal codes is due to the existence of non-diagonal interference terms in the grammian matrix. In the case of conventional QO-STBC, there is a presence of pair wise decoding rather than the individual symbol decoding at the receiver end; these non-diagonal terms are responsible for the creation of interference from the neighboring elements which makes the system more complex. To overcome these drawbacks, a new paradigm is introduced for three transmit antennas which can bring out improved diversity gain and less decoding complexity by making a diagonal detection matrix. Analysis has been done for conventional QO-STBC3, RARQOSTBC3 and Taha3 scheme for three transmit antenna and different receive antenna configurations. Study over different channels and simulation results for the different order of PSK modulation shows that the RAR-QOSTBC3 outperforms the conventional QO-STBC3 and Taha3 scheme regarding bit error rate.
\end{abstract}

Keywords: BER, Linear Zero-Forcing Decoder, M-PSK, QO-STBC, RAR-QOSTBC, Taha

\section{Introduction}

In past decades, wireless communication trade has known for their significant growth. In wireless communication, analysis becomes more challenging to get reliable transmission between ends because of the presence of random paths. A signal travels from a transmitter antenna to an intended receiver through different paths having different obstructions in their path or affected by certain physical parameters which ultimately subjects to performance degradation known as fading. Fading coefficients relevant to different existing wireless paths between both ends are independent and uncorrelated to each other. Limited spreading power at the transmitter side and scarce frequency bandwidth are the resource constraints present in the wireless communication. Subjective to the constraints of wireless scenario, a new concept of diversity techniques came into existence which is having performance enhancing capabilities by combating fading effects and without sacrificing the frequency bandwidth and spreading power at the transmitter end. As the name itself specified, diversity accounts to provide the multiple copies of the same data at the receiver side. Reliability of communication at the recipient will improve because the probability that some of the replicas will not undergo deep fade also increases. Diversity gain depends upon the number of transmitters and receivers deployed in the system because mathematically the number of transmitters multiply with some receivers gives the total number of available paths or diversity order. To get the benefits of both diversity techniques and channel coding at the transmitter end results in a new approach called space-time block codes. The basis of STBC can be defined as the transmission of ordered redundancy of informa-

${ }^{*}$ Author for correspondence 
tion, which leads to performance improvement of the system by achieving different types of gains like array gain, spatial diversity gain, etc. Interference reduction can also be accomplished by increasing the spatial separation between different users. The main concern in wireless systems is to get the minimum BER on given SNR value.

Recent work has concentrated on transmit diversity schemes which mean using multiple antennas at the transmitter side to enhance the performance of the setup. This scheme is more economical to the society because extra equipments are added to the base station rather than at remote station. First, transmit diversity scheme is introduced by Alamouti ${ }^{1}$ which is also regarded as first Space-time block code. Full rate and full diversity are provided by Alamouti space time block code with the contour of $2^{\star} 1$ that means using two Tx. antennas and one $\mathrm{Rx}$. antenna. It can be simply generalized to higher configurations by changing the number of receiver antennas to get more diversity order. So the basic transmit diversity scheme is effective with all the required parameters of wireless communication as it can provide improved error performance and data rate of the overall system. To make a system more reliable, there is a need to increase the number of transmit antenna. Tarokh introduced a new paradigm for transmit diversity by using multiple antennas at the transmitter side ${ }^{2}$. These code structures are constructed by orthogonal designs. Maximum diversity gain is achieved by using linear ML decoding at the recipient end. The reason of simple linear decoding at the recipient is the presence of a diagonal decoding matrix. According to Tarokh, an orthogonal code structure which is containing complex terms in it further cannot achieve rate one for larger than two transmit antennas. The maximum rate of 3/4 (i.e. conveyance of three symbols over four-time slots) can be achieved with complex orthogonal codes for 3 and 4 transmit antennas. For reliable communication a new concept of code structure is given in ${ }^{3}$ where channel conditions are also considerable i.e. fading channel. Diversity gain and performance gain are given by the minimum rank and minimum determinant of the matrices respectively created by encoding symbol sequence. Keeping wireless communication constraints into mind, full encoding algorithms, as well as decoding algorithms, are specified in ${ }^{4}$. O-STBC's are known as the optimal codes to achieve minimum BER and maximum signal to noise ratio i.e. SNR 5 .

Jafarkhani introduced new channel codes recognized as quasi-orthogonal space-time block codes which are having characteristics contradictory to the statement of Tarokh i.e. full rate can be achieved for configuration higher than two transmit antennas having complex terms in their code structure ${ }^{6}$. The design principle of this code structure depends upon the quasi-orthogonal design where orthogonality is achieved with the pairing of columns rather than the single column. Disadvantages of these code structures are partial diversity gain and more decoding load at the receiver. The reason of more decoding complexity in QO-STBC is the interference provided by neighboring signals. With the increase in the modulation order, this complexity will also increase and leads to transmission delay. If the data rate is increased beyond the data rate granted by orthogonality, then the code structure is no more orthogonal. So to reduce this non-orthogonality, a noval rate one code design is given in $^{7}$. Existing QO-STBC can be modified in such a way that can provide more flexibility for certain mechanisms in wireless communication like the selection of antenna, water-filling, etc. are given in ${ }^{-}$. These code structures are easy to design because of having low encoding/decoding complexity. In a decoder of QO-STBC, pairs of the symbol are taken at once for decoding that leads to increase in the computational load. Even decoding of pairs of the symbol cannot be done with simple linear decoding. So to make a decoding simpler, a new code structure is introduced in ${ }^{9}$. Next encoding/decoding processes for different existing QO-STBC structures can be modified to get a linear decoding and to make a system more robust $\mathrm{t}^{\mathrm{10}}$. Application of symmetric property on detection matrix can help us to get a diagonal detection matrix and value of the interference terms can be decreased by using the concept of antenna shuffling to obtain maximum SNR and minimum BER ${ }^{11}$. RAR-QOSTBC scheme is designed for

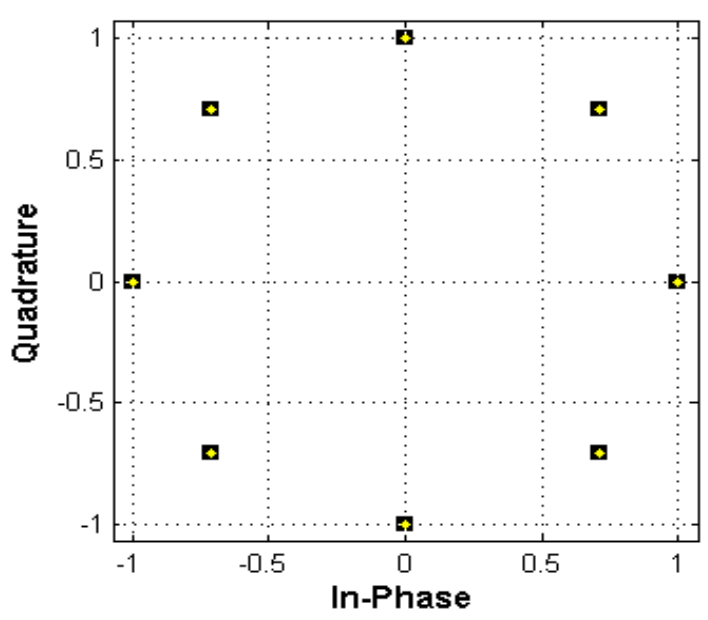

Figure 1. Constellation diagram for 8-PSK. 
four transmit antennas in ${ }^{12}$ by using linear algebra concepts.

In this paper, an RAR-QOSTBC3, QOSTBC3 and Taha3 scheme are given for three transmit antennas. The RAR-QOSTBC3 scheme is proposed by using various concepts like reflection and rotations $s^{13,14}$. By removing interference terms from a detection matrix of four transmit antenna further it can be extended to three transmit antennas $\frac{15,16}{}$. Simulation comparison has done for conventional QO-STBC3, RAR-QOSTBC3 and Taha3 scheme for three transmit antenna over the different order of PSK modulation.

\section{Modulation Technique and Channels}

In this paper, the different order of PSK modulation i.e. M-PSK scheme is used to modulate the symbols so that it can travel through the wireless medium over different channels like AWGN channel and Rayleigh fading channel. Description for the same is given below-

\subsection{M-PSK Modulation Scheme}

In digital communication, PSK modulation scheme is a most widely used in growing fields of data communication because of its efficient transmission as compared to other modulation schemes. In phase-shift keying modulation scheme, information is transmitted by varying the phase of the carrier signal or reference signal according to the main message signal. Each form of PSK modulation uses a fixed number of phases, and each phase corresponds to one particular pattern of binary digits. PSK symbols follow the below Equation

$S_{m}(t)=\sqrt{\frac{2 E_{b}}{T_{b}}} \cos \left\{2 \pi f_{c} t+\frac{2 \pi(m-1)}{M}\right\} \mathbf{m}=1,2, \ldots, \mathbf{M} \& 0<t<T_{b}$

where $E_{b}$ is the signal energy per symbol, $S_{m}$ is the modulated signal $f_{c}$ is the carrier frequency and $T_{b}$ is the symbol duration. The phase of the reference signal will change according to the phase factor $\theta$ given below-

$$
\theta=\frac{2 \pi(m-1)}{M} \mathrm{~m}=1,2 \ldots, \mathrm{M}
$$

PSK modulation formats are having circular constellation because every symbol is having the same amount of energy i.e. energy of symbols in PSK modulation does not depend upon the phases assigned to particular binary bit patterns as shown in Figure 1.

\subsection{AWGN Channel}

AWGN channel is the basic standard which is commonly used to analyze the behavior of any system over different modulation scheme. Some assumptions are considered in the AWGN channel which are given below-

- Amplitude-frequency response of channel is flat for all frequencies.

- Phase-frequency response of channel is linear for all frequencies.

AWGN channel does not consider any obstacles in the channel i.e. line of sight communication. Impairments are present in the AWGN channel analysis only because of summation of white Gaussian noise with constant power spectral density at the recipient end. Therefore, the received signal can be given as-

$$
y(t)=m(t)+n(t)
$$

where $m(t)$ is the transmitted signal, $n(t)$ is the white Gaussian noise with zero mean and constant power spectral density $\frac{N_{0}}{2}$.

\subsection{Rayleigh Channel}

In the case of multipath propagation, fading can be described by Rayleigh fading model. This model uses the statistical approach to evaluate the characteristics of propagation. This channel model is particularly used in the scenario when there is no dominant path available between both ends for communication. In this model, all the trappings related to channel mechanisms like reflection, diffraction and scattering are noticeable. Timevarying nature of each multipath signal is represented by Rayleigh distribution.

Probability density function of Rayleigh distribution is expressed as-

$$
p(r)= \begin{cases}\frac{r}{\sigma^{2}} e^{\left(-\frac{r^{2}}{2 \sigma^{2}}\right)} & (0 \leq r \leq \infty) \\ 0 & (r<0)\end{cases}
$$

where $\sigma^{2}$ is the time-average power of the received signal.

\section{Conventional QO-STBC3 Scheme}

Conventional QO-STBC containing complex terms in their code structures are demonstrated by Jafarkhani $\underline{6}$ 


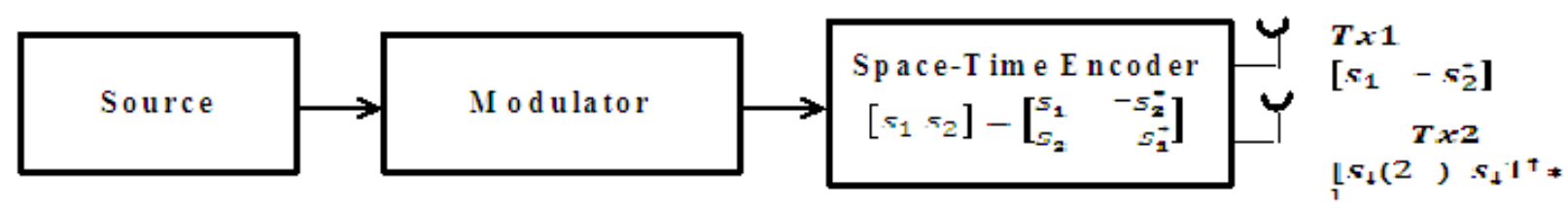

Figure 2. Block diagram of Alamouti Space-time encoder.

which are having the characteristics of achieving full rate for higher configurations i.e. for greater than two transmit antenna. We can also say that QO-STBC is the extended version of Alamouti ${ }^{1}$ scheme because the sub-symbol terms present in the QO-STBC code structure contains the Alamouti sequence for transmitting symbols. So, the encoding sequence of Alamouti scheme is given in Figure 2.

Therefore, the symbol transmission matrix of Alamouti scheme is given as-

$$
S_{12}=\left[\begin{array}{rr}
s_{1} & s_{2} \\
-s_{2}^{*} & s_{1}^{*}
\end{array}\right]
$$

and QOSTBC encoding matrix is expressed as

$S=\left[\begin{array}{cc}S_{12} & S_{34} \\ -S_{34}^{*} & S_{12}^{*}\end{array}\right]=\left[\begin{array}{cccc}s_{1} & s_{2} & s_{3} & s_{4} \\ -s_{2}^{*} & s_{1}^{*} & -s_{4}^{*} & s_{3}^{*} \\ -s_{3}^{*} & -s_{4}^{*} & s_{1}^{*} & s_{2}^{*} \\ s_{4} & -s_{3} & -s_{2} & s_{1}\end{array}\right]$

Let $\widetilde{H}=\left[\begin{array}{llll}h_{1} & h_{2} & h_{3} & h_{4}\end{array}\right]^{T}$ is a complex channel coefficients, $\tilde{Y}=\left[\begin{array}{llll}r_{1} & r_{\mathbf{2}} & r_{3} & r_{\mathbf{4}}\end{array}\right]^{T}$ is a received signal vector therefore the received signal can be expressed as

$$
\widetilde{Y}=S \widetilde{H}+\widetilde{N}
$$

where $\widetilde{N}=\left[n_{1} n_{2} n_{3} n_{\mathbf{4}}\right]^{T}$ is an additive white Gaussian noise added at the receiver. The channel matrix can be generated by applying complex conjugate operations on the second and the third row of the encoding matrix is given as

$$
H=\left[\begin{array}{cccc}
h_{1} & h_{2} & h_{3} & h_{4} \\
h_{2}^{*} & -h_{1}^{*} & h_{4}^{*} & -h_{3}^{*} \\
h_{3}^{*} & h_{4}^{*} & -h_{1}^{*} & -h_{2}^{*} \\
h_{4}^{*} & -h_{3} & -h_{2} & h_{1}
\end{array}\right]
$$

Channel matrix for three transmit antenna can be derived from the above matrix by putting the value of $\boldsymbol{h}_{\mathbf{4}}=\mathbf{0}$ that can be expressed as

$$
H=\left[\begin{array}{cccc}
h_{1} & h_{2} & h_{3} & 0 \\
h_{2}^{*} & -h_{1}^{*} & 0 & -h_{2}^{*} \\
h_{3}^{*} & 0 & -h_{1}^{*} & -h_{2}^{*} \\
0 & -h_{3} & -h_{2} & h_{1}
\end{array}\right]
$$

So, the encoding matrix of conventional QO-STBC for three transmit antenna can be written as

$$
S=\left[\begin{array}{ccc}
s_{1} & s_{2} & s_{3} \\
-s_{2}^{*} & s_{1}^{*} & -s_{4}^{*} \\
-s_{3}^{*} & -s_{4}^{*} & s_{1}^{*} \\
s_{4} & -s_{3} & -s_{2}
\end{array}\right]
$$

Hence, the received signal is given as

$$
Y=H s+N
$$

where, $s=\left[\begin{array}{llll}s_{1} & s_{2} & s_{3} & s_{\mathbf{4}}\end{array}\right]^{T}$ and

$N=\left[n_{1} n_{\mathbf{2}}^{*} n_{3}^{*} n_{\mathbf{4}}\right]^{T}$. The Grammian matrix or detection matrix, which is used for detection at the recipient end, is expressed as

$$
G=H^{H} H
$$

Linear processing at the receiver can only be possible if the detection matrix is a diagonal matrix. On the other hand, detection matrix of QO-STBC contains non-diagonal terms that are known as interference terms. Detection matrix of conventional QO-STBC for three transmit antenna is expressed as

$$
\begin{aligned}
& G=D=H^{H} \cdot H=\left[\begin{array}{cccc}
a & 0 & 0 & b \\
0 & a & -b & 0 \\
0 & -b & a & 0 \\
a & 0 & 0 & a
\end{array}\right]
\end{aligned}
$$

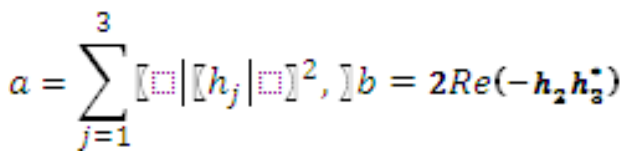

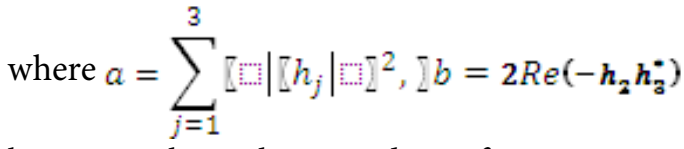

are known as channel gain and interference terms respectively.

\section{Taha3 Scheme}

For a real symmetric matrix $D$ as given by (13), there exists a real unitary matrix $Q$ such that 
$D_{n}=Q^{T} D Q$

where $Q$ is a real unitary matrix and ${ }^{D}$ is a diagonal matrix.

Let the unitary matrix is given as

$$
Q=\left[\begin{array}{cccc}
\frac{1}{\sqrt{2}} & 0 & 0 & -\frac{1}{\sqrt{2}} \\
0 & \frac{1}{\sqrt{2}} & -\frac{1}{\sqrt{2}} & 0 \\
0 & \frac{1}{\sqrt{2}} & \frac{1}{\sqrt{2}} & 0 \\
\frac{1}{\sqrt{2}} & 0 & 0 & \frac{1}{\sqrt{2}}
\end{array}\right]
$$

By using Singular Value Decomposition, desired diagonal matrix $D_{n}$ can be generated by pre and postmultiplying $D$ with $Q^{T}$ and $Q$ respectively

$D_{n}=Q^{T} D Q=\left[\begin{array}{cccc}a+b & \mathbf{0} & \mathbf{0} & \mathbf{0} \\ \mathbf{0} & a-b & \mathbf{0} & \mathbf{0} \\ \mathbf{0} & \mathbf{0} & a+b & \mathbf{0} \\ \mathbf{0} & \mathbf{0} & \mathbf{0} & a-b\end{array}\right]$

New channel matrix $H_{n}$ can be derived from Equation (16) and expressed as

$$
\begin{aligned}
& D_{n}=Q^{T} D Q \\
& =Q^{T} H^{T} H Q \\
& =(H Q)^{T} \cdot(H Q)
\end{aligned}
$$

where $H$ is taken from Equation (9). Therefore, the new channel matrix is $H_{n}=H Q$

$H_{n}=\left(\frac{1}{\sqrt{2}}\right) *\left[\begin{array}{cccc}h_{1} & h_{2}+h_{3} & -h_{2}+h_{3} & -h_{1} \\ h_{2}^{*}-h_{3}^{*} & -h_{1}^{*} & h_{1}^{*} & -h_{2}^{*}-h_{3}^{*} \\ h_{3}^{*}-h_{2}^{*} & -h_{1}^{*} & -h_{1}^{*} & -h_{2}^{*}-h_{3}^{*} \\ h_{1} & -h_{2}-h_{3} & -h_{2}+h_{3} & h_{1}\end{array}\right]$

TAHA encoding matrix corresponding to new channel matrix for 3 transmit antenna can be expressed as

$$
S_{n}=\left(\frac{1}{\sqrt{2}}\right) *\left[\begin{array}{ccc}
s_{1}-s_{4} & s_{2}-s_{3} & s_{2}+s_{3} \\
-s_{2}^{*}+s_{3}^{*} & s_{1}^{*}-s_{4}^{*} & -s_{1}^{*}-s_{4}^{*} \\
-s_{2}^{*}-s_{3}^{*} & -s_{1}^{*}-s_{4}^{*} & s_{1}^{*}-s_{i}^{*} \\
s_{1}+s_{4}^{*} & -s_{2}-s_{3} & -s_{2}+s_{3}
\end{array}\right]
$$

\section{RAR-QOSTBC3 Scheme}

Orthogonal matrix transformation methods like Householder transformations or reflectors, which defines the reflection across the plane that is orthogonal to a unit normal vector ' $u$ '. Matrix format for the same can be expressed as

$$
\begin{aligned}
& Q=I-2 u u^{T} \\
& \text { let } u=\left[\begin{array}{ll}
\cos \theta & -\sin \theta
\end{array}\right]^{T} \\
& \text { and }|| u \|_{2}=1 \\
& H_{w}=I-2 u u^{T}=\left[\begin{array}{ll}
1 & 0 \\
0 & 1
\end{array}\right]-2\left[\begin{array}{c}
\cos \theta \\
-\sin \theta
\end{array}\right][\cos \theta-\sin \theta] \\
& =\left[\begin{array}{ll}
1-2 \cos ^{2} \theta & 2 \cos \theta \sin \theta \\
2 \cos \theta \sin \theta & 1-2 \sin ^{2} \theta
\end{array}\right] \\
& =\left[\begin{array}{cc}
-\cos 2 \theta & \sin 2 \theta \\
\sin 2 \theta & \cos 2 \theta
\end{array}\right]
\end{aligned}
$$

Flow Chart 1. Algorithm for proposed scheme.

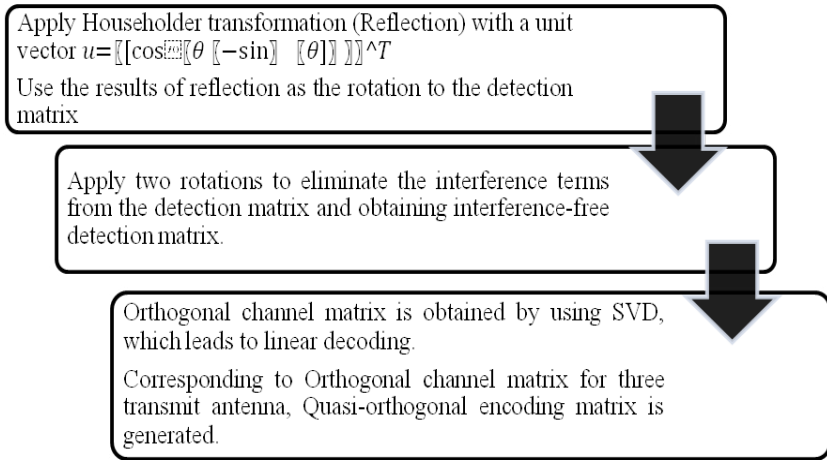

Equation (22) can be used as a rotation with one-dimensional reflection. Rotation is the matrix computation method which is commonly used to remove the particular terms from the given matrix. Elimination of interference terms from the detection matrix (i.e. nondiagonal terms) will be done by applying two rotations. Interference term ${ }^{b}$ as given in (11) can be eliminated during first rotation from index $D_{14}$ and $D_{41}$

$$
\begin{aligned}
& R_{p}=\left[\begin{array}{cccc}
-\cos 2 \theta & \mathbf{0} & \mathbf{0} & \sin 2 \theta \\
\mathbf{0} & \mathbf{1} & \mathbf{0} & \mathbf{0} \\
\mathbf{0} & \mathbf{0} & \mathbf{1} & \mathbf{0} \\
\sin 2 \theta & \mathbf{0} & \mathbf{0} & \cos 2 \theta
\end{array}\right] \\
& \text { where } \theta=\frac{\pi}{8} ; \text { Rest of the interferen }
\end{aligned}
$$
given in (11) can be eliminated during second rotation from index $D_{23}$ and $D_{32}$

$$
R_{q}=\left[\begin{array}{cccc}
1 & 0 & 0 & 0 \\
0 & -\cos 2 \theta & \sin 2 \theta & 0 \\
0 & \sin 2 \theta & \cos 2 \theta & 0 \\
0 & 0 & 0 & 1
\end{array}\right]
$$

Linear detection matrix can be generated by using singular value decomposition method which results in interference free detection matrix.

$$
D_{n}=Q^{T} D Q
$$




$$
\begin{aligned}
& D_{n}=R_{q}^{T} \cdot R_{p}^{T} \cdot D \cdot R_{p} \cdot R_{q} \\
& R_{p} \cdot R_{q}=\left[\begin{array}{cccc}
-\frac{1}{\sqrt{2}} & 0 & 0 & \frac{1}{\sqrt{2}} \\
0 & -\frac{1}{\sqrt{2}} & \frac{1}{\sqrt{2}} & 0 \\
0 & \frac{1}{\sqrt{2}} & \frac{1}{\sqrt{2}} & 0 \\
\frac{1}{\sqrt{2}} & 0 & 0 & \frac{1}{\sqrt{2}}
\end{array}\right] \\
& R_{q}^{T} \cdot R_{p}^{T}=\left[\begin{array}{cccc}
-\frac{1}{\sqrt{2}} & 0 & 0 & \frac{1}{\sqrt{2}} \\
0 & -\frac{1}{\sqrt{2}} & \frac{1}{\sqrt{2}} & 0 \\
0 & \frac{1}{\sqrt{2}} & \frac{1}{\sqrt{2}} & 0 \\
\frac{1}{\sqrt{2}} & 0 & 0 & \frac{1}{\sqrt{2}}
\end{array}\right] \\
& D_{n}=\left[\begin{array}{cccc}
a-b & \mathbf{0} & \mathbf{0} & \mathbf{0} \\
\mathbf{0} & a+b & \mathbf{0} & \mathbf{0} \\
\mathbf{0} & \mathbf{0} & a-b & \mathbf{0} \\
\mathbf{0} & \mathbf{0} & \mathbf{0} & a+b
\end{array}\right]
\end{aligned}
$$

Hence, the linearly decodable diagonal matrix can be represented as $D_{n}=H_{n}^{H} \cdot H_{n}$. Therefore, the new channel matrix $H_{n}$ derived from the Equation (29) will be given by the following derivation-

$$
\begin{aligned}
& D_{n}=R_{q}^{T}\left(R_{p}^{T} \cdot D \cdot R_{p}\right) R_{q} \\
& =\left(R_{q}^{T} \cdot R_{p}^{T}\right) \cdot H^{H} H \cdot\left(R_{p} \cdot R_{q}\right) \\
& =\left(R_{q} R_{p}\right)^{T} \cdot H^{H} H \cdot\left(R_{p} R_{q}\right) \\
& =\left(H \cdot R_{p} R_{q}\right)^{H}\left(H \cdot R_{q} R_{p}\right) \\
& D_{n}=H_{n}^{H} \cdot H_{n} \\
& H_{n}=H \cdot R_{p} \cdot R_{q}
\end{aligned}
$$

After computing Equation (32), new channel matrix $H_{n}$ can be expressed as

$$
H_{n}=\left(\frac{1}{\sqrt{2}}\right) *\left[\begin{array}{cccc}
-h_{1} & -h_{2}+h_{3} & h_{2}+h_{3} & h_{1} \\
-h_{2}^{*}-h_{3}^{*} & h_{1}^{*} & -h_{1}^{*} & h_{2}^{*}-h_{3}^{*} \\
-h_{2}^{*}-h_{3}^{*} & -h_{1}^{*} & -h_{1}^{*} & -h_{2}^{*}+h_{3}^{*} \\
h_{1} & -h_{2}+h_{3} & -h_{2}-h_{3} & h_{1}
\end{array}\right]
$$

Therefore the noval RAR-QOSTBC symbol matrix corresponding to channel matrix $H_{n}$ for three transmit antennas can be given as

$$
S_{n}=\left(\frac{1}{\sqrt{2}}\right) *\left[\begin{array}{ccc}
-s_{1}+s_{4}^{*} & -s_{2}+s_{3}^{*} & s_{2}+s_{3} \\
s_{2}^{*}-s_{3}^{*} & -s_{1}^{*}+s_{4}^{*} & -s_{1}^{*}-s_{4}^{*} \\
-s_{2}^{*}-s_{3}^{*} & -s_{i}^{*}-s_{4}^{*} & -s_{1}^{*}+s_{4}^{*} \\
s_{1}+s_{4}^{*} & -s_{2}-s_{3} & s_{2}-s_{3}
\end{array}\right]
$$

Now, the above channel matrix is an orthogonal matrix which allows linear decoding at the receiver and encoding matrix is still quasi-orthogonal in nature.

Hence, the received signal is given by following-

$$
Y=H_{n} s+N
$$

where $\mathrm{N}$ is the Gaussian noise with zero mean.

\section{Zero-Forcing Equalizer}

Zero-forcing equalizer is a linear detection technique to restore the information at the receiver. It is the efficient method because it uses the inverse of the frequency response of channel at the receiver so that combination of both channel and equalizer provides flat frequency response and linear phase. i.e. if $\mathrm{F}(\mathrm{f})$ is the frequency response of channel and $\mathrm{C}(\mathrm{f})$ is the frequency response of the equalizer produced by taking the inverse of the frequency response of channel i.e. $1 / F(f)$, then it will results to $\mathrm{F}(\mathrm{f})^{\star} \mathrm{C}(\mathrm{f})=1$. An estimate signal $\hat{s}$ equivalent to $\mathrm{s}$ can be found as-

$$
\hat{s}=\left(H^{H} H\right)^{-1} H^{H} Y
$$

As given in the above expression there is a need to calculate the inverse of detection matrix. So it will be easy to calculate the inverse of detection matrix if it contains only diagonal elements. Therefore, we are computing detection matrix in such a way that results in the diagonal matrix.

\section{Simulation Results}

In this section, the performance comparison of conventional QO-STBC3, Taha3 scheme and RAR-QOSTBC3 for three transmit antenna over AWGN channel has shown in Figure 3. Simulation has displayed for different orders of PSK modulation. Figure 3(a) displays the performance plots of conventional QO-STBC3, Taha3 scheme and RAR-QOSTBC3 over 8-PSK modulation. Taha3 scheme is performing better than conventional QO-STBC3 up to a particular value of SNR and as the SNR value increases, conventional QO-STBC3 scheme outperforms the Taha3 scheme $e^{10}$. Now, the code structure RAR-QOSTBC3 for three transmit antenna outperforms from both conventional QO-STBC3 and Taha3. In the case of RAR-QOSTBC, linear decoding leads to less complexity as well as better performance. But in the case of Taha3, complexity is decreased, but performance is not better than conventional QO-STBC as the SNR keeps on increasing i.e. in RAR-QOSTBC. The efficient rotation 
matrix is used which further eliminates all the non-diagonal or interference terms from the detection matrix. One more concept of receiver diversity can be seen in the plots which means as the numbers of the receiver are increasing then it ultimately leads to increase in the performance gain because the numbers of random paths from the transmitter to the receiver are increasing.

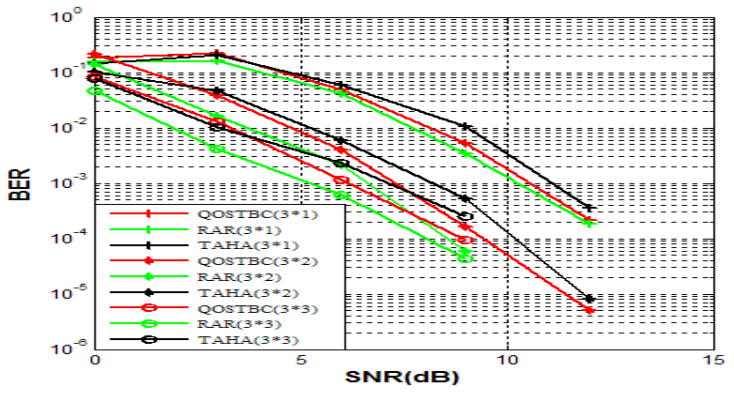

(a)

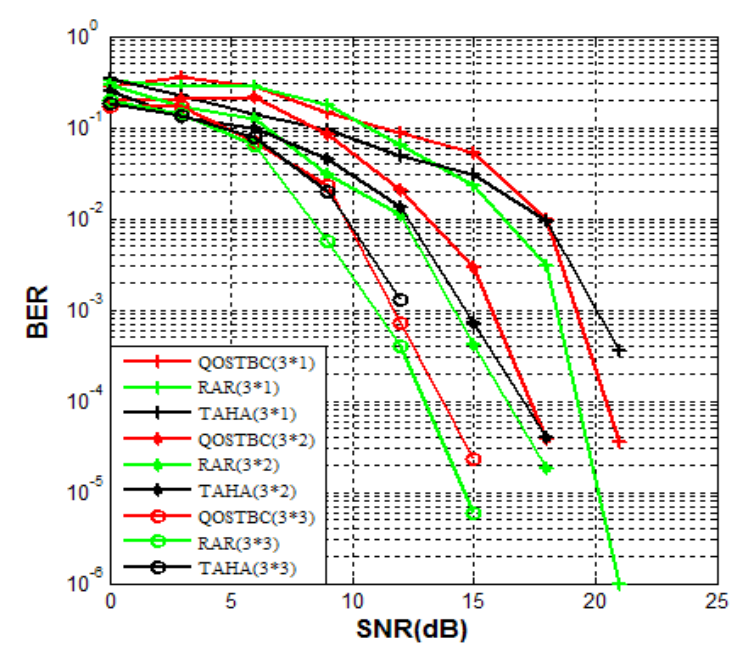

(b)

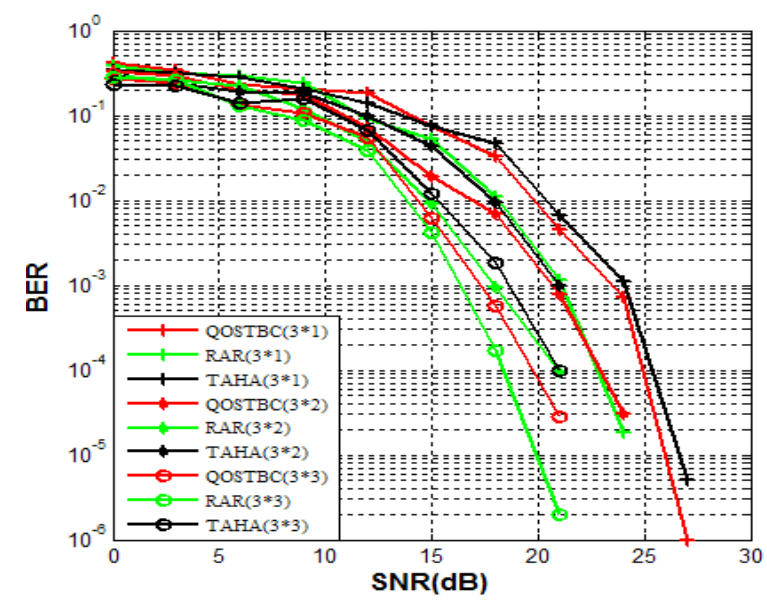

(c)

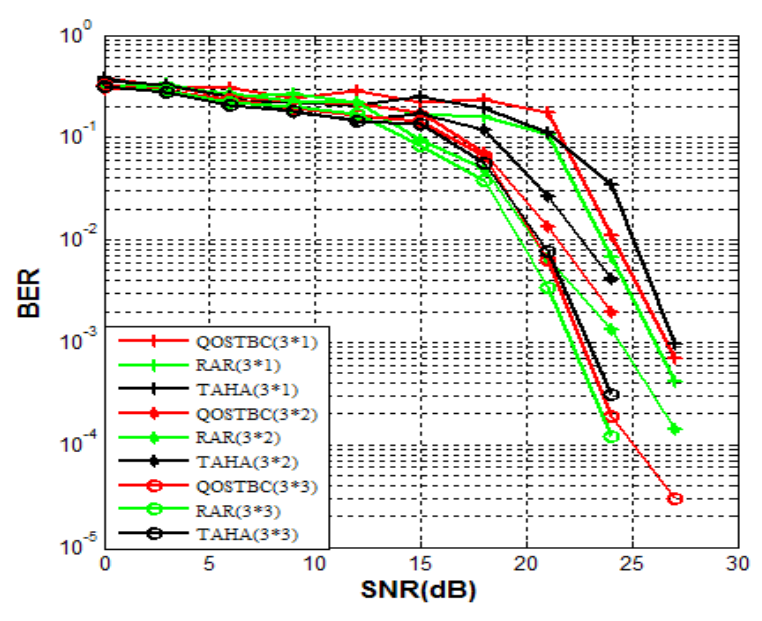

(d)

Figure 3 (a-d): BER vs. SNR plots for Conventional QOSTBC, Taha and RAR-QOSTBC employing M-PSK schemes over AWGN channel (a) 8-PSK (b) 16-PSK (c) 32PSK (d) 64-PSK.

It is also clear from the plots that SNR required to achieve BER of $10^{-3}$ is decreasing from $10.75 \mathrm{~dB}$ to 6.2 $\mathrm{dB}$ for conventional QO-STBC3 as the receiver antennas are increasing from one to three. Similar effects can be seen in the case of Taha3 scheme and RAR-QOSTBC3. For the configuration of three transmit and one receive antenna conventional QO-STBC3 requires SNR of 10.75 $\mathrm{dB}$ whereas Taha scheme requires SNR of $11.25 \mathrm{~dB}$ and RAR-QOSTBC requires SNR of $10.30 \mathrm{~dB}$ So, there is a coding gain in the RAR-QOSTBC3 scheme as compared to conventional QO-STBC3 and Taha3 scheme. In Figure 3(b)-(d), the performance comparison of these three schemes has done for higher levels of PSKmodulation. As the order of modulation increases, then the SNR required for same antenna configuration will also increase. Numerical values of required SNR for particular BER are given in Table 1.

Now, the simulation results have shown for the performance of conventional QO-STBC3, Taha3 and RAR-QOSTBC3 scheme for three transmit antenna over Rayleigh fading environment. One assumption has made that the channel is quasi-static in nature i.e. channel parameters may remain consistent for one entire frame and receiver knows all about the channel conditions (Channel state information). Same amount of power is allocated to all the transmit antennas.

Figure 4(a-d) displays the simulation results for performance of conventional QO-STBC3, Taha scheme 
Table 1. SNR required achieving BER of $10^{-3}$ over AWGN channel

\begin{tabular}{|c|c|c|c|c|c|c|c|c|c|}
\hline \multicolumn{10}{|c|}{ SNR(dB) REQUIRED TO ACHIEVE BER OF $10^{-3}$} \\
\hline \multicolumn{4}{|c|}{$\begin{array}{l}\text { QOSTBC(with different antenna } \\
\text { configuration) }\end{array}$} & \multicolumn{3}{|c|}{$\begin{array}{l}\text { TAHA(with different antenna } \\
\text { configuration) }\end{array}$} & \multicolumn{3}{|c|}{$\begin{array}{l}\text { RAR(with different antenna } \\
\text { configuration) }\end{array}$} \\
\hline $\begin{array}{l}\text { Modulation } \\
\text { level }\end{array}$ & $3 T x^{\star} 1 R x$ & $3 T x^{\star} 2 R x$ & $3 T x^{\star} 3 R x$ & $3 T x^{\star} 1 R x$ & $3 T x^{\star} 2 R x$ & $3 T^{*} 3 R x$ & $3 T x^{\star} 1 R x$ & $3 T x^{\star} 2 R x$ & $3 T x^{*} 3 R x$ \\
\hline 8-PSK & 10.75 & 7.5 & 6.2 & 11.25 & 8.25 & 7.2 & 10.3 & 6.75 & 5.2 \\
\hline 16-PSK & 19 & 14.7 & 11.75 & 20 & 15.75 & 12 & 18.3 & 14.25 & 11 \\
\hline 32-PSK & 23.5 & 20.75 & 17.2 & 24 & 21 & 18.5 & 21 & 18 & 16.5 \\
\hline 64-PSK & 26.6 & 24.9 & 22.7 & 27 & 25.5 & 23 & 26.10 & 24.5 & 22.2 \\
\hline
\end{tabular}

and RAR-QOSTBC3 for three transmit antenna over Rayleigh fading channel for 8, 16, 32, 64 PSK modulation respectively. RAR-QOSTBC3 for three transmit antenna is performing better as compared to conventional QO-STBC3 and Taha3 scheme for all the cases. In the case of three transmit and one receive antenna for 8PSK modulation scheme, conventional QO-STBC3 requires SNR of $17.5 \mathrm{~dB}$ whereas Taha3 scheme requires SNR of $18.5 \mathrm{~dB}$ and RAR-QOSTBC3 requires SNR of $16.5 \mathrm{~dB}$ So, it is clear that RAR-QOSTBC3 is having coding gain of $2 \mathrm{~dB}$ as compared to the conventional QO-STBC3 and coding gain of $1 \mathrm{~dB}$ as compared to the Taha3 scheme. Similarly, for the higher order of modulation format, there is a coding gain in case of the RAR-QOSTBC3 scheme as compared to conventional QO-STBC3 and Taha3 scheme. This proves that the proposed RAR-QOSTBC3 scheme is more robust to channel estimating errors as compared to already existing schemes. All the numerical values of SNR required for BER of $10^{-3}$ are given in Table 2 .

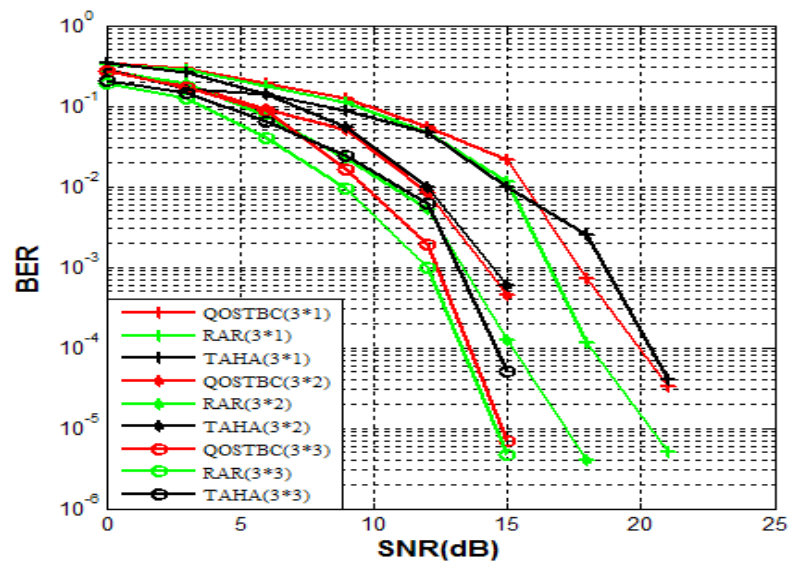

(a)

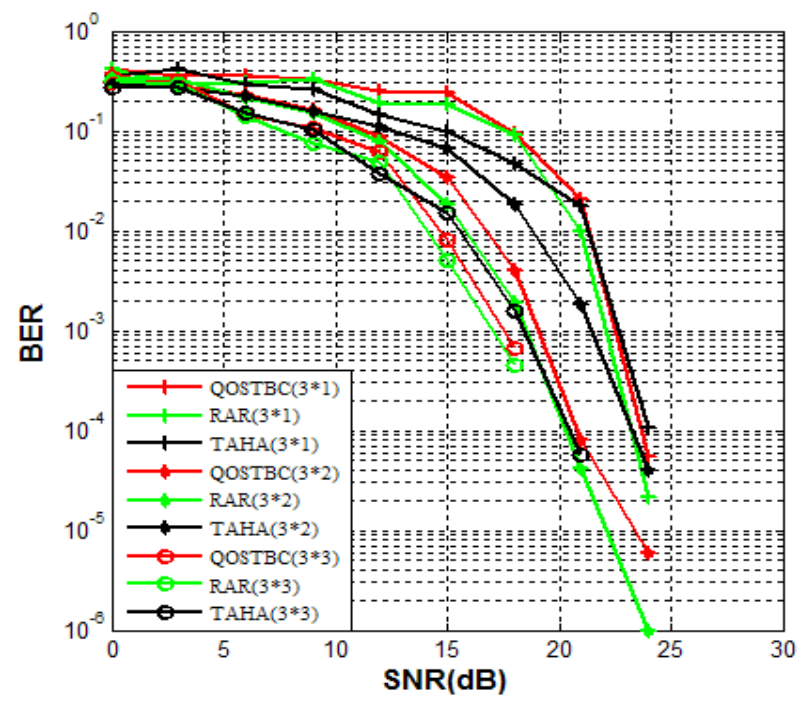

(b)

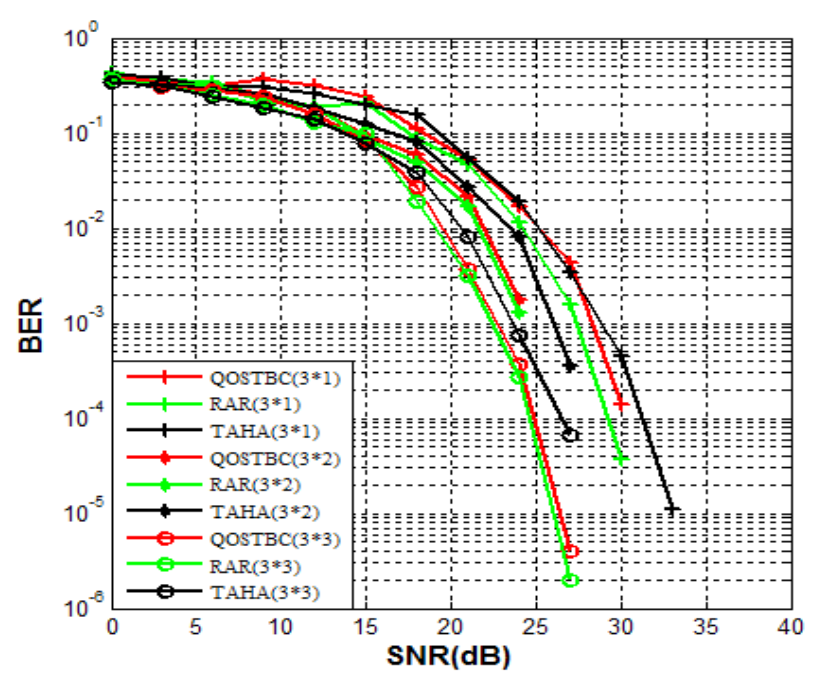

(c) 
Table 2. SNR required achieving BER of $10^{-3}$ over Rayleigh channel

\begin{tabular}{|c|c|c|c|c|c|c|c|c|c|}
\hline \multicolumn{10}{|c|}{ SNR(dB) REQUIRED TO ACHIEVE BER OF $10^{-3}$} \\
\hline \multicolumn{4}{|c|}{$\begin{array}{l}\text { QOSTBC(with different antenna } \\
\text { configuration) }\end{array}$} & \multicolumn{3}{|c|}{$\begin{array}{l}\text { TAHA(with different antenna } \\
\text { configuration) }\end{array}$} & \multicolumn{3}{|c|}{$\begin{array}{l}\text { RAR(with different antenna } \\
\text { configuration) }\end{array}$} \\
\hline $\begin{array}{l}\text { Modulation } \\
\text { level }\end{array}$ & $3 \operatorname{Tx}^{\star} 1 \mathrm{Rx}$ & $3 T x^{\star} 2 R x$ & $3 T x^{\star} 3 R x$ & $3 \operatorname{Tx}^{\star} 1 \mathrm{Rx}$ & $3 T x^{\star} 2 R x$ & $3 T x^{\star} 3 R x$ & $3 T x^{\star} 1 R x$ & $3 T x^{\star} 2 R x$ & $3 T x^{\star} 3 R x$ \\
\hline 8-PSK & 17.5 & 14.1 & 12.3 & 18.5 & 14.3 & 13 & 16.5 & 13.2 & 12 \\
\hline 16-PSK & 22.5 & 19 & 17.5 & 22.6 & 21.5 & 18.35 & 22 & 18.5 & 17 \\
\hline 32-PSK & 28.4 & 24.4 & 22.6 & 29 & 26 & 23.6 & 27.5 & 24 & 22.4 \\
\hline 64-PSK & 34.4 & 33 & 29 & 36.5 & 33 & 29.35 & 33.4 & 31.2 & 29 \\
\hline
\end{tabular}

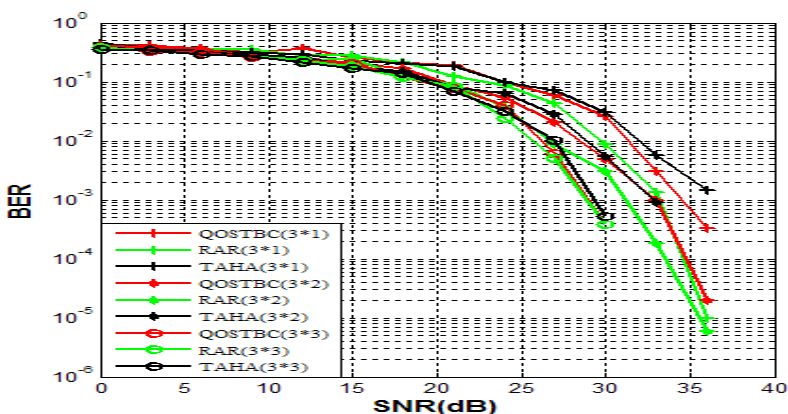

(d)

Figure 4. BER vs. SNR plots for conventional QOSTBC, Taha and proposed QOSTBC employing M-PSK schemes over Rayleigh channel (a) 8-PSK (b) 16-PSK (c) 32-PSK (d) 64-PSK.

\section{Conclusion}

In this paper, linearly decodable RAR-QOSTBC3 for three transmit antenna is proposed which is performing better than proposed conventional QO-STBC 3 and Taha3 scheme for the same configuration of the antenna. By the use of certain methods of matrix computation like reflection and rotation, we can construct a diagonal detection matrix for three transmit antenna further orthogonal channel matrix for same configuration, and corresponding quasi-orthogonal symbol transmission matrix can be generated. Linear decoding is achieved because of the removal of non-diagonal terms from the detection matrix, which results in decreasing decoding complexity and increasing coding gain with respect to other schemes like Taha3 and conventional QO-STBC3. In Taha3 scheme, decoding complexity is decreased, but there is no coding gain as the comparison to the RAR-QOSTBC3 scheme. The BER versus SNR curve converges at more rates in case of AWGN channel where as in Rayleigh channel; the rate of convergence is low because of the presence of environment effects. Therefore, the overall coding gain in case of AWGN channel is more than the coding gain in Rayleigh channel. The further work can be performed on other configuration of transmit antenna with different modulation schemes over diverse channels.

\section{References}

1. Alamouti SM. A simple transmit diversity technique for wireless communications. IEEE Journal on Selected Areas in Communications. 1998 Oct; 16:1451-8.

2. Tarokh V, Seshadri N, Calderbank A. Space-time codes for high data rate wireless communication: Performance criterion and code construction. IEEE Transactions on Information Theory. 1998; 44:744-65.

3. Tarokh V, Jafarkhani H, Calderbank AR. Space-time block codes from orthogonal designs. IEEE Transactions on Information Theory. 1999 Jul; 45:1456-67.

4. Tarokh V, Jafarkhani H, Calderbank AR. Space-time block coding for wireless communication: Performance results. IEEE Journal on Selected Areas in Communications. 1999; 17:451-60.

5. Ganesan G, Stoica P. Space-time block codes: Maximum SNR approach. IEEE Transactions on Information Theory. 2001; 45:1650-6.

6. Jafarkhani H. A quasi-orthogonal space-time blocks code. IEEE Transactions on Communications. 2001 Jan; 49:1-4.

7. Tirkkonen O, Boariu A, Hottinen A. Minimal non-orthogonality rate 1 space-time block code for $3+$ Tx antennas. IEEE 6th International Symposium on Spread Spectrum Techniques and Applications. 2000; 2:429-32.

8. Badic B, Rupp M, Weinrichter H. Quasi-orthogonal spacetime block codes: Approaching Optimality. The 13th European Signal Processing Conference (EUSIPCO); 2005.

9. Park U, Lim K, Li J. A novel QO-STBC scheme with linear decoding for three and four transmit antennas. IEEE Communications Letters. 2008; 12:868-70. 
10. Taha ZQ, Farraj AK. Efficient decoding for generalized quasi-orthogonal space-time blocks codes. Wireless Personal Communications. 2013; 68:1731-43.

11. Sharma V, Sharma S. Noval linear decodable QO-STBC for four transmits antennas with transmits antenna shuffling. Wireless Personal Communications. 2015; 82:47-59.

12. Chugh S, Kansal L. Performance comparison of linearlydecodable RAR-QOSTBC and conventional QO-STBC for M-PSK modulation. Optik-International Journal for Light and Electron Optics. 2016; 127:5374-9.

13. Golub GH, Van Loan CF. Matrix Computations. 3rd ed. Johns Hopkins University Press; 1996.
14. Lang S. Linear Algebra. $2^{\text {nd }}$ ed. New York, NY: Springer; 1987.

15. Manikandan C, Neelamegam P, Divya E. OFDM techniques for MIMO-OFDM system: A review. Indian Journal of Science and Technology. 2015 Sep; 8(22). Doi no:10.17485/ijst/2015/v8i22/79103

16. Kasiselvanathan M, Sathish Kumar N. BER performance analysis and comparison for large scale MIMO receiver. Indian Journal of Science and Technology. 2015 Dec; 8(35). Doi no:10.17485/ijst/2015/v8i35/81073 